\title{
Where Is the Catheter? Identifying Misplacement of a Dialysis Catheter
}

\author{
Xiao-xia Zhang ${ }^{\text {a Hong-xiu Chen }}{ }^{\mathrm{b}}$ Chang Liuc Xiu-ying Hu ${ }^{\mathrm{d}}$ \\ ${ }^{a}$ Department of Breast Surgery, West China Hospital, Sichuan University, Chengdu, PR China; ${ }^{b}$ West China School

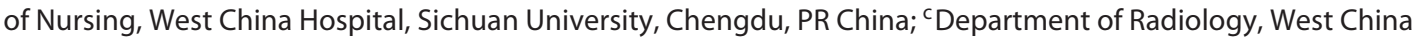 \\ Hospital, Sichuan University, Chengdu, PR China; dWest China School of Medicine/West China Hospital, Sichuan \\ University, Chengdu, PR China
}

Dear Editor,

We read with great interest the paper authored by Li Cavoli et al. [1]. The paper describes a case having a hemodialysis catheter misplaced into hemiazygos vein (HAV). In Li Cavoli and Schillaci's case, the computer tomography (CT) images showed that the catheter was on the right side very close to the spine, which had the least radiographic parallax error on the chest X-ray (CXR) image. Therefore, the vertebrae and costovertebral joint can be used as the landmark to precisely evaluate the catheter location. The CXR and CT images showed that the catheter originated at the right root of the neck, then decreased along T1-4 close to the right side of the spine, and then slightly deviated to the left at T5 level, finally terminated near the fifth sternocostal joints (Fig. 1A, G) [1]. After a comprehensive review of the reported cases with misplaced central venous catheter (CVC) into azygos vein $(\mathrm{AV})$, the anatomy of $\mathrm{AV}$ and $\mathrm{HAV}$ (Fig. 1A-J) [1-8], we conclude that the authors misinterpreted the findings in the case; the catheter was most probably misplaced into the AV rather than into the $\mathrm{HAV}$ as the authors declared.

AV usually starts from the posterior to inferior vena cava, ascends along the right side of spine to T3 or T4 level, where $73.4 \%$ of the AV arches forward and enters into the superior vena cava (Fig. 1E, J) [8]. The HAV is formed on the left side of spine from the lower 3 left posterior intercostal veins, ascending to the T7 level below where $84.2 \%$ of the HAV influxes into AV (Fig. 1J) [8]. Thus, there are 2 inconsistencies between the post-anterior radiographic findings in Li Cavoli and Schillaci's case and the anatomical relationship of AV and HAV. First, HAV is located on the left side of the inferior 1/3 part of spine, but in Li Cavoli and Schillaci's case, the catheter is positioned on the right side of the superior 1/3 part of spine (Fig. 1A). Second, in Li Cavoli and Schillaci's case, the catheter terminates at the T5 level on the right side of the spine, from where a distant gap of 2-5 vertebrae exists to where the catheter enters into HAV (Fig. 1A, J). Therefore, the catheter could not enter into HAV as Li Cavoli and Schillaci declared.

The AV terminated in a diameter ranged from 5.0 to $12.2 \mathrm{~mm}$ in a cadaver research [8] and from 4.6 to $16.0 \mathrm{~mm}$ in living human detected by CT [9], resulting in CVC misplacement inadvertently. In reported cases having a CVC mis- placed into $\mathrm{AV}$, the radiographic manifestation presented by CXR and CT images were in high consistence with those presented by Li Cavoli and Schillaci's case (Fig. 1A-D, G-I) $[1-4,6,7]$. The anatomical characteristics of AV arch and its opening into superior vena cava would demonstrate some special radiographic features, which could alert us of a potential catheter misplacement [10]. The abnormal radiographic characteristics could usually be a hump of the catheter at the T4 or T5 level, or an abnormal shape twisting to either the right side or the left side of the spine.

\section{Disclosure Statement}

The authors have no conflicts of interest to declare.

\section{Funding Sources}

This work was supported by research grant from the Department of Science and Technology Research Projects of the Sichuan Province, PR China (grant number 2019YFS0382).

\section{KARGER}

(c) 2019 S. Karger AG, Basel
Xiu-ying $\mathrm{Hu}$

West China School of Medicine/West China Hospital

Sichuan University

No.37 Guo Xue Street, Chengdu, Sichuan Province 610041 (PR China)

E-Mail huxiuying@scu.edu.cn 

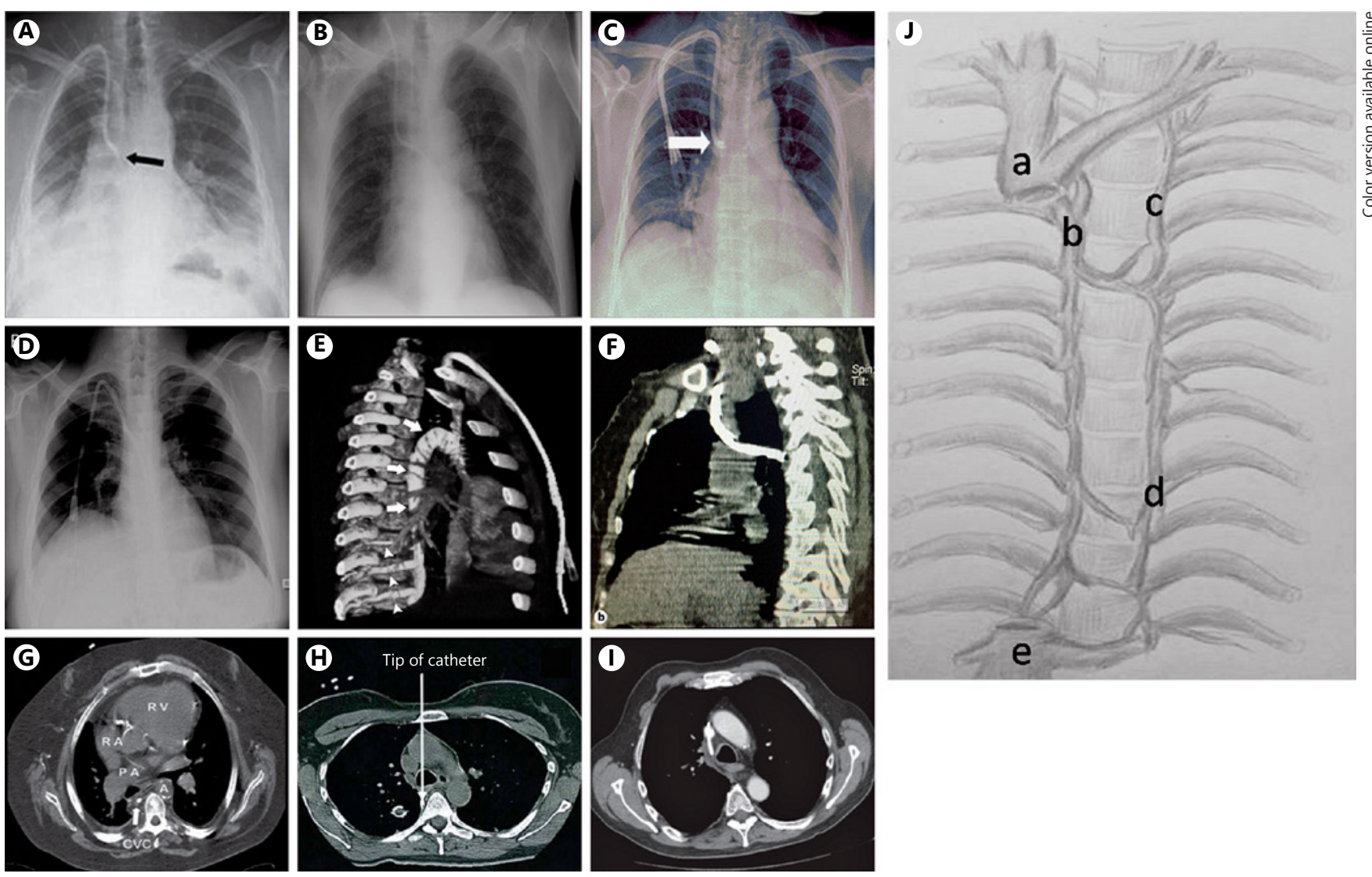

Fig. 1. Radiographic image of catheter misplaced into the AV and anatomy of the AV system. A, G CXR and CT images in Li Cavoli and Schillaci's case: The catheter originated at the right root of the neck, then decreased along T1-4 closing to the right side of the spine, and then slightly deviated to the left at T5 level, finally terminated closing to the fifth sternocostal joints. B-D, F, H, and I Catheters misplaced into AV: In the cases reported having a CVC misplaced into AV, the CXR and CT images presented were in high consistence with those presented by Li Cavoli and Schillaci's case. E A dilated AV with retrograde contrast medium demonstrating the radiographic anatomical relationship of the AV and spine on lateral image. F A catheter misplaced into AV on lateral image. J Anatomy of the AV system. a Superior vena cava. b AV. c Left superior intercostal vein. d HAV. e Inferior vena cava. The Figure 1 A-I is reprinted with permission.

\section{References}

1 Li Cavoli G, Schillaci O, Servillo F, Zagarrigo C, Li Cavoli TV, Palmeri M, et al. Malposition of the central venous catheter in the hemiazygos vein. Blood Purif. 2016;42(2): $168-9$.

2 Calviño J, Bravo J, Martínez L, Millán B, Pulpeiro JR. Recognizing misplacement of a dialysis catheter in the azygos vein. Hemodial Int. 2013 Jul;17(3):455-7.

3 Mou LJ, Wang SH, Li QH, Zhang RS, Pan NZ, Wang JF. Malpositioning of a tunneled hemodialysis catheter into the azygos vein: a case report and literature review. Blood Purif. 2017;44(2):108-9.

4 Pua U. Imaging teaching case. Radiographic features of malpositioning of a hemodialysis catheter in the azygos vein. Am J Kidney Dis. 2010 Feb;55(2):395-8.

5 Demant AW, Rattunde H, Abderhalden S, Von Vigier R, Wolf RW. Pitfall in pediatric dialysis: malposition of a dialysis catheter mimicking azygos continuation syndrome. J Vasc Access. 2007 Oct-Dec;8(4):302-4.

6 Markovich MB. Central venous catheter tip placement: determination of posterior malposition - a case study. J Assoc Vasc Access. 2006;11(2):85-9.

7 Andrews MH, Chisholm BD. Spontaneous migration of a portacath into the azygos vein with subsequent development of a tracheoazygos fistula. J Med Imaging Radiat Oncol. 2015 Apr;59(2):200-3.
8 Kutoglu T, Turut M, Kocabiyik N, Ozan $\mathrm{H}$, Yildirim M. Anatomical analysis of azygos vein system in human cadavers. Rom J Morphol Embryol. 2012;53(4):1051-6.

9 Tatar I, Denk CC, Celik HH, Oto A, Karaosmanoglu DA, Ozdemir BM, et al. Anatomy of the azygos vein examined by computerized tomography imaging. Saudi Med J. 2008 Nov; 29(11):1585-8

10 Haygood TM, Brennan PC, Ryan J, Yamal JM, Liles L, O'Sullivan P, et al. Central venous line placement in the superior vena cava and the azygos vein: differentiation on posteroanterior chest radiographs. AJR Am J Roentgenol. 2011 Apr;196(4): $783-7$. 